\title{
Philosophiques
}

\section{Conceptualiser les troubles mentaux chez les enfants et les adolescents}

\section{Christian Perring}

Volume 33, numéro 1, printemps 2006

Philosophie et psychopathologie

URI : https://id.erudit.org/iderudit/012947ar

DOI : https://doi.org/10.7202/012947ar

Aller au sommaire du numéro

\section{Éditeur(s)}

Société de philosophie du Québec

ISSN

0316-2923 (imprimé)

1492-1391 (numérique)

Découvrir la revue

Citer cet article

Perring, C. (2006). Conceptualiser les troubles mentaux chez les enfants et les adolescents. Philosophiques, 33(1), 65-79. https://doi.org/10.7202/012947ar
Résumé de l'article

J'explore de façon critique la supposition du DSM et de théoriciens tels que Wakefield et Gert selon laquelle les troubles mentaux doivent être attribués à un individu plutôt qu'à un groupe de personnes. Cette supposition est particulièrement problématique en pédopsychiatrie où le système familial est très souvent au centre de l'attention clinique. Il y a bien sûr des éléments de preuve substantiels indiquant que certains troubles mentaux des individus sont causés par leurs relations avec les autres et que leur guérison est grandement facilitée en traitant le groupe, tel que la famille, comme un tout. Malgré cela, il y a eu beaucoup moins de travail conceptuel visant à définir ce que cela pourrait être pour un couple, une famille ou un autre groupe que de se voir attribuer un trouble mental. Pour traiter de cette question, j'utilise un débat entre Wakefield (2000) et Murphy et Woolfolk (2000) sur la question de savoir s'il fait partie du concept de trouble mental que celui-ci soit causé par une dysfonction interne de la personne. Je discute aussi de la proposition faite par Bolton (2000) d'abandonner complètement le concept de trouble mental et d'utiliser plutôt le concept, plus large, de problème de santé mentale. Je soutiens qu'en fin de compte le caractère individuel des troubles mentaux ne constitue pas une vérité conceptuelle a priori, et qu'il faut faire intervenir des considérations pragmatiques pour décider s'il est utile de nous limiter à une telle définition ou si nous pourrions être mieux servis par une définition plus extensive. Je fais le lien avec le pragmatisme et je soutiens qu'une approche pluraliste non réductive est particulièrement appropriée en pédopsychiatrie.
Tous droits réservés @ Société de philosophie du Québec, 2006
Ce document est protégé par la loi sur le droit d'auteur. L’utilisation des services d'Érudit (y compris la reproduction) est assujettie à sa politique d'utilisation que vous pouvez consulter en ligne.

https://apropos.erudit.org/fr/usagers/politique-dutilisation/ 


\title{
Conceptualiser les troubles mentaux chez les enfants et les adolescents
}

\author{
CHRISTIAN PERRING \\ Dowling College \\ cperring@yahoo.com
}

\begin{abstract}
RÉSUMÉ. - J'explore de façon critique la supposition du DSM' et de théoriciens tels que Wakefield et Gert selon laquelle les troubles mentaux doivent être attribués à un individu plutôt qu'à un groupe de personnes. Cette supposition est particulièrement problématique en pédopsychiatrie où le système familial est très souvent au centre de l'attention clinique. Il y a bien sûr des éléments de preuve substantiels indiquant que certains troubles mentaux des individus sont causés par leurs relations avec les autres et que leur guérison est grandement facilitée en traitant le groupe, tel que la famille, comme un tout. Malgré cela, il y a eu beaucoup moins de travail conceptuel visant à définir ce que cela pourrait être pour un couple, une famille ou un autre groupe que de se voir attribuer un trouble mental. Pour traiter de cette question, j'utilise un débat entre Wakefield (2000) et Murphy et Woolfolk (2000) sur la question de savoir s'il fait partie du concept de trouble mental que celui-ci soit causé par une dysfonction interne de la personne. Je discute aussi de la proposition faite par Bolton (2000) d'abandonner complètement le concept de trouble mental et d'utiliser plutôt le concept, plus large, de problème de santé mentale. Je soutiens qu'en fin de compte le caractère individuel des troubles mentaux ne constitue pas une vérité conceptuelle a priori, et qu'il faut faire intervenir des considérations pragmatiques pour décider s'il est utile de nous limiter à une telle définition ou si nous pourrions être mieux servis par une définition plus extensive. Je fais le lien avec le pragmatisme et je soutiens qu'une approche pluraliste non réductive est particulièrement appropriée en pédopsychiatrie.
\end{abstract}

ABSTRACT. - I critically explore the assumption of the DSM and theorists such as Wakefield and Gert that mental disorder must be attributed to an individual rather than a group of people. This assumption is especially problematic in child and adolescent psychiatry where very often the focus of clinical attention is the family system. There is of course substantial evidence that some mental disorders of individuals are caused by their relationships with other people and that their recovery is greatly facilitated by treating the group, such as a family, as a whole. However, there has been much less conceptual work on defining what it might be for a couple, family or other group to itself be attributed a mental disorder. To address this issue, I employ a debate between Wakefield (2000), Murphy and Woolfolk (2000) on whether it is part of the concept of a mental disorder that it is caused by an internal malfunction of a person. I also discuss the proposal of Bolton (2000) that we do away with the concept of mental disorder altogether and instead use a broader concept of mental health problem. I argue that ultimately it is not an a priori conceptual truth that mental disorders are individual, and we need to bring in pragmatic considerations to decide whether it is helpful to restrict ourselves to such a definition or whether we could be better

1. N. D.T. : Le Diagnostic and Statistical Manual de l'American Psychiatric Association.

PHILOSOPHIQUES 33/1 — Printemps 2006, p. 65-80 
served by a more expansive definition. I link this to the philosophical view of pragmatism and argue that a pluralistic nonreductive approach is especially appropriate in child and adolescent psychiatry.

\section{Introduction}

Bien qu'en pédopsychiatrie la question de la nosologie n'ait pas été au centre de beaucoup de recherches, un travail considérable a déjà été fait, et dans le cadre de ce travail, des débats sur les catégories diagnostiques ont eu lieu. Dans ce champ disciplinaire, on a souvent exprimé de l'insatisfaction au sujet des différentes éditions du Diagnostic and Statistical Manual de l'American Psychiatric Association, et une publication de 1996 sur les troubles de l'enfance, par le Group for the Advancement of Psychiatry, souvent citée pour son importance, mentionne qu'il faut «mettre l'accent sur les méandres du développement normal au sein d'un spectre allant de la normalité à la pathologie, ainsi que sur les significations multiples des symptômes » (Schowalter, 2000, p. 468). En psychiatrie théorique, en philosophie et en éthique médicale, beaucoup de travail a été fait récemment sur le concept de trouble mental (voir Perring, 2002, pour une revue de la littérature). Le but de ce débat est d'établir une analyse des troubles mentaux qui soit fondée, utile et claire, et qui permettrait de clore le débat sur les catégories controversées de troubles mentaux telles que l'homosexualité, les paraphilies, la psychopathie et différentes formes de dépendance. Cette analyse s'appliquerait à tous les troubles mentaux, y compris ceux des enfants et des adolescents. Il y a certainement eu un débat à propos de la légitimité de certaines sortes de diagnostics en pédopsychiatrie ; certains critiques ont suggéré, par exemple, que le $\mathrm{TDAH}^{2}$ est surdiagnostiqué et que les jeunes garçons sont par nature hyperactifs, ou que le changement dans la durée d'attention des enfants est une réaction à des changements plus généraux dans notre culture " en mode rafale " (['rapid-fire' culture] ; DeGrandpre, 1999). D'autres préconisent une approche nettement plus médicalisée en ce qui concerne le diagnostic du TDAH. Par exemple, Hudziak soutient une approche taxonomique qui « facilite la sélection correcte des sujets pour les études d'identification des gènes » de façon à " améliorer notre capacité à identifier les facteurs de risque génétiques " (2002, p. 211). Il conclut ainsi son argument :

Par exemple, les enfants avec TDAH pourraient dorénavant être décrits comme TDAH-Gène récepteur de dopamine 4 (7), TDAH-Gène transporteur de dopamine (10) et TDAH-Gène récepteur de dopamine 3. Si le potentiel de la nouvelle génétique médicale se réalise, les traitements pourraient fort bien varier selon le génotype (Hudziak, 2002, p. 227).

Le cas des enfants et des adolescents est important pour le débat sur l'analyse des troubles mentaux parce qu'il offre un défi intéressant aux approches

2. N. D. T. : Trouble déficitaire de l'attention avec hyperactivité. 
standards. Avant de considérer la question principale, nous devons examiner une caractéristique particulière de la pédopsychiatrie. Bien que de nombreux troubles mentaux se retrouvent autant chez les enfants que chez les adultes, ils présentent souvent des groupes de symptômes assez différents. Par exemple, même si le DSM-IV-TR (American Psychiatric Association, 2000) ne donne pas de critères de diagnostics différents pour identifier un épisode de dépression majeure chez les adultes et chez les enfants, il explique que, lorsque les enfants vivent un épisode de dépression majeure, ils sont particulièrement susceptibles de présenter des symptômes tels que des plaintes à caractère somatique, de l'irritabilité et un retrait social, mais sont peu susceptibles de présenter un retard psychomoteur, de l'hypersomnie et des délires (American Psychiatric Association, 2000, p. 354). La ICD-103 énumère certains troubles d'anxiété spécifiques aux enfants et aux adolescents (Organisation mondiale de la santé, 1992) bien que le DSM-IV-TR ne le fasse pas. Même si un trouble mental est diagnostiqué selon les mêmes critères chez les adultes et chez les enfants, il y a des différences quant à la façon dont les symptômes sont compris. Qu'une émotion, ou l'expression de celle-ci, soit appropriée ou bien pathologique chez une jeune personne dépend beaucoup de l'âge de cette dernière. Par exemple, un accès de rage peut être normal chez un jeune enfant, mais anormal chez un adolescent (U.S. Department of Health and Human Services, 1999, p. 123). De même, lors du diagnostic des manies, il est probable que ce que l'on considère comme des idées de grandeur ou de la distractivité chez les adultes pourrait être considéré comme un comportement normal chez les enfants ou les adolescents. La catégorisation des troubles mentaux des enfants et des adolescents doit donc être extrêmement reliée à notre compréhension de leur maturation psychologique. Étant donné que les troubles mentaux sont actuellement classifiés par groupes de symptômes plutôt que par causes sous-jacentes, nous pourrions nous demander quelle justification il y a à traiter deux cas présentant des symptômes forts différents comme deux cas de la même espèce de trouble mental. Mais cela n'est pas un problème majeur pour la classification psychiatrique, puisque le fait qu'il soit pratique de regrouper plusieurs ensembles de symptômes sous la catégorie d'un même trouble mental constitue une justification suffisante pour ce faire, à tout le moins lorsque cette classification ne pose pas de problème évident.

\section{La maladie mentale comme condition familiale}

Nous pouvons aller au cœur de la question en considérant un énoncé du chapitre "Les enfants et la santé mentale » dans le rapport de 1999 du US Surgeon General sur la santé mentale :

La prise en considération des principes développementaux améliore notre compréhension de la maladie mentale chez les enfants et les adolescents en réconciliant le concept de trouble mental comme état stable, ou condition, avec le

3. N. D. T. : La International Classification of Diseases, de l'Organisation mondiale de la santé, dixième édition. 
développement continu de l'enfant. Selon ces principes, un trouble mental résulte de l'interaction d'un enfant avec son environnement. Ainsi, ce n'est souvent pas seulement chez l'enfant que se trouve la maladie mentale. À l'intérieur du cadre conceptuel et du langage intégratif des neurosciences, le trouble mental est une " propriété émergente » résultant du contact avec l'environnement (U.S. Department of Health and Human Services, 1999, p. 136).

Ce qui est remarquable dans cette affirmation sur la nature du trouble mental, c'est qu'elle va à l'encontre de la plupart des théories standards considérant celui-ci comme la caractéristique fondamentale d'une personne, ou parfois du cerveau d'une personne. Par exemple, Gert et al. (1997, p. 101) insistent pour que la maladie ${ }_{g}^{4}$ (un terme un peu plus général que " affection ») soit une condition attribuable à un individu.

Bien sûr, le rapport du Surgeon General ne se veut pas un travail qui concerne le débat philosophique sur la nature de la maladie mentale, et il ne serait donc pas sage de considérer que ses définitions font autorité. L'objectif des définitions du rapport est plus probablement de mettre l'accent sur le fait que la plupart des troubles mentaux des enfants et des adolescents sont le résultat d'une situation familiale ou sociale particulière, ou à tout le moins qu'ils doivent être compris dans le cadre de cette situation, que de soutenir une thèse métaphysique quant à la maladie. L'idée mérite néanmoins que l'on cherche à voir si la compréhension des troubles mentaux, caractéristique de la pédopsychiatrie, pose problème pour le débat philosophique en ce qui concerne leur définition. En particulier, on peut se demander s'il est conceptuellement possible que les troubles mentaux, de familles ou de groupes, ne soient pas responsables des troubles mentaux de leurs membres individuels.

Borduin et al. (1999, p. 498) mettent l'accent sur le fait qu' " il y a maintenant des éléments de preuve substantiels suggérant que les troubles émotionnels et comportementaux des enfants sont souvent liés à des problèmes relationnels avec leur famille, leurs pairs et leur système scolaire ». De plus, ils établissent un lien explicite entre cette observation et certaines critiques incisives des systèmes existants de classification des troubles mentaux des enfants et des adolescents, puisque DSM-IV et ICD-10, tout en admettant l'existence des problèmes relationnels, ne leur accordent que peu d'attention et leur assignent un rôle négligeable dans le diagnostic des troubles mentaux. Il existe plusieurs modèles théoriques pour comprendre les problèmes relationnels, entre autres les théories de l'apprentissage social, des systèmes familiaux et une approche de thérapie multisystémique. Dans ces modèles, l'unité fondamentale est la famille ou un système plus large, plutôt que les individus qui constituent cette unité. Il peut y avoir une relation de renforcement mutuel entre les problèmes mentaux des enfants et les attitudes et comportements de leurs

4. Note du traducteur : Gert donne au terme anglais relativement inusité « malady » une définition technique particulière (voir plus bas). Pour le distinguer de "illness" (que nous traduisons par «maladie »), nous utilisons l'indice ${ }_{\mathrm{g}}$ : " maladie $_{\mathrm{g}}$ ". 
parents. Par exemple, les enfants qui reçoivent peu d'affection parentale courent le risque de développer des troubles dépressifs, et certains parents peuvent devenir émotionnellement moins affectueux envers leurs enfants déprimés (voir Borduin, 1999, p. 504). Plusieurs théoriciens ont soutenu qu'il s'agit là d'une approche fondamentalement différente pour la compréhension des troubles mentaux. Par exemple, Henggler et Borduin disent que « les théoriciens des systèmes et les thérapeutes familiaux ont modifié la façon dont un grand nombre de professionnels de la santé mentale conceptualisent et traitent les problèmes chez les enfants et les adolescents" (1990, p. vii). De même, Cummings et al. soutiennent que "le "trouble" n'est pas nécessairement le fait d'un individu, mais peut dans certains cas être dû au contexte social »; ils suggèrent que de tels concepts promettent " une compréhension plus complète du développement humain » (2000, p. 20).

\section{Distinguer les troubles individuels des troubles familiaux}

Avant de traiter cette question, nous devons faire une distinction capitale entre ce que j'appelle les problèmes « causalement relationnels » et les problèmes " intrinsèquement relationnels ». Cette distinction est rarement faite, lorsqu'elle est faite, dans la littérature sur la théorie des systèmes. Peut-être qu'elle importe peu pour les approches de traitement et que les médecins ne sentent donc pas la nécessité de la faire. Il est néanmoins évident que plusieurs des problèmes dont discutent les théoriciens des systèmes sont causalement relationnels. Ces problèmes sont les problèmes individuels d'un enfant ou d'un adolescent qui sont causés et maintenus par le reste de la famille ou d'un groupe plus large. Par exemple, dans le cadre d'une discussion de la dépendance aux drogues et à l'alcool chez les adolescents, Milton Trapold explique qu' "il n'y a pas de raison de croire que la famille cause la dépendance aux drogues et à l'alcool de quelque façon que ce soit ", mais par la suite il affirme que le soutien familial est essentiel à la guérison de l'adolescent (Trapold, dans Henggler et Borduin, 1990, p. 268). Le manque de soutien suffisant de la part de la famille peut faire que le problème persiste chez le patient. Néanmoins, la dépendance est un trouble du patient. De façon plus générale, il est évident que les troubles mentaux individuels peuvent avoir des causes génétiques, interpersonnelles et sociales. En ce qui nous concerne, la question est de savoir s'il peut y avoir des troubles mentaux intrinsèquement relationnels, qui ne peuvent être attribués qu'à des personnes en relation avec d'autres personnes et qui ne se réduisent pas à deux personnes ayant des problèmes purement individuels. On pourrait même penser aux troubles intrinsèquement relationnels comme à des troubles de groupes plutôt que, ou peut-être en plus, des troubles individuels.

Il est important de noter que plusieurs chercheurs croient qu'il y a des troubles intrinsèquement relationnels. Reis et Emde (2002), dans leur article "Relational Disorders Are Psychiatric Disorders ", en sont un exemple notoire. Néanmoins, bien que ces chercheurs fassent une démonstration empirique de la façon dont les problèmes relationnels sont à la fois réels et 
traitables, ils n'abordent pas la question conceptuelle, à savoir si ces problèmes devraient être classifiés comme des troubles mentaux. Même les auteurs dont les concepts philosophiques sont plus raffinés n'aboutissent à de telles conclusions qu'avec des arguments très généraux. Jensen et Hoagwood, par exemple, soutiennent explicitement qu'en ce qui concerne les enfants " tous les troubles "mentaux" proviennent des communautés, de l'entourage et des familles » (1997, p. 238). Néanmoins, leur argument repose surtout sur la variabilité culturelle du concept de personne. De tels arguments sont provocateurs, mais si nous reconnaissons que la psychiatrie est relative à la culture, et que c'est sur la culture occidentale que nous nous concentrons, alors les pratiques des autres cultures sont d'une pertinence limitée lorsqu'il s'agit de guider nos propres pratiques.

\section{Le modèle de la dysfonction préjudiciable et ses critiques}

Deux articles récents sur la nature des troubles mentaux ont accordé une attention minutieuse aux problèmes relationnels. Pour expliquer cette discussion, il sera nécessaire de résumer quelque peu le débat qui précède l'examen des questions relationnelles. Jerome Wakefield a longuement défendu, dans une série d'articles récents, la thèse selon laquelle les troubles mentaux se définissent par deux conditions à la fois nécessaires et suffisantes : un trouble doit être le résultat de l'insuffisance d'un ou plusieurs mécanismes internes dans l'exécution d'une fonction. Cette définition des troubles mentaux est souvent appelée "dysfonction préjudiciable » [harmful dysfunction].

Murphy et Woolfolk (2000) soutiennent que certains troubles mentaux, y compris les troubles déficitaires de l'attention avec hyperactivité (TDAH), pourraient ne pas être le résultat de quelque dysfonction « interne " et, si on les évalue du point de vue de l'évolution, pourraient même mener à une plus grande capacité de reproduction dans certaines circonstances environnementales (2000, p. 244). Malgré cela, ils avancent que même si cette théorie spéculative de la psychologie évolutionniste était correcte, dans le monde contemporain, on considérerait quand même le comportement associé au TDAH comme étant un trouble, alors qu'il ne l'est pas selon l'analyse de Wakefield. Murphy et Woolfolk citent aussi la théorie de l'apprentissage social et les approches cognitivo-comportementales des problèmes relationnels pour soutenir qu'il y aurait des troubles mentaux où le problème n'est pas dû à quelque dysfonction interne de l'apprentissage, mais plutôt à un environnement problématique qui fournit les mauvais données, lesquelles entraînent les mauvais réponses.

Dans son exposé, Wakefield (2000) insiste sur le fait que ces arguments ne sont pas de bons contre-exemples pour son analyse des troubles mentaux. Il s'oppose à l'affirmation selon laquelle il est évident que le TDAH doit compter comme un trouble mental, soutenant que nous pourrions découvrir de nouvelles informations qui changeraient notre évaluation de son statut. Par exemple, s'il était démontré que le TDAH est une condition qui augmente la 
capacité de reproduction, on pourrait changer d'opinion quant au fait qu'il doive compter comme un trouble mental (Wakefield, 2000, p. 259). Il fait aussi remarquer qu'il y a déjà une controverse à ce sujet et que cela ne constitue donc pas un contre-exemple très solide pour son analyse en termes de dysfonctions préjudiciables.

Wakefield soutient que son analyse peut satisfaire aux exigences des cas de la théorie de l'apprentissage. Il cite des données selon lesquelles les problèmes relationnels ne sont effectivement pas nécessairement considérés comme des troubles mentaux par les professionnels de la santé, et dit que la théorie cognitivo-comportementale n'a jamais prétendu que ces problèmes étaient des troubles mentaux. Par exemple, les cliniciens jugent qu'un adolescent dont les symptômes satisfont aux critères du DSM quant aux troubles de comportement n'a pas de problème si son comportement peut être expliqué en termes de réactions apprises normales à des facteurs environnementaux (2000, p. 261). De plus, Wakefield affirme que l'explication de la genèse d'un problème comportemental en tant que condition apprise est compatible avec la compréhension du problème en tant que résultat d'une dysfonction interne. Il utilise le bogue de l'an 2000 comme exemple : les ordinateurs auraient pu mal fonctionner lorsque nous sommes passés au nouveau millénaire même si aucun mécanisme interne n'était " défectueux ". Le mauvais fonctionnement aurait plutôt été causé par une donnée d'entrée pour laquelle l'ordinateur n'était pas conçu. De même façon, soutient Wakefield, les mécanismes d'apprentissage des enfants pourraient ne pas être défectueux, mais, par exemple, s'ils ont pour environnement une famille très dysfonctionnelle, le processus pourrait néanmoins mal fonctionner pour des raisons que nous qualifierions d'internes (pp. 262-4). Ainsi, Wakefield croit que sa théorie admet le fait qu'il s'agit de cas de troubles mentaux.

Cet échange entre les différents auteurs devrait nous amener à considérer avec plus d'attention ce qui compte comme étant la raison interne d'un trouble et ce qui compte comme étant un problème relationnel. Murphy et Woolfolk ne soutiennent pas que les familles peuvent avoir des troubles intrinsèques, mais ils soutiennent bel et bien qu'un individu peut avoir un trouble sans qu'aucune dysfonction interne ne soit en cause. Wakefield défend sa vision des choses en insistant sur le fait que les cas qu'on lui propose comme contre-exemples de sa définition ne lui sont pas fatals parce qu'ils peuvent être correctement décrits comme ceux d'individus ayant des dysfonctions internes ; il utilise donc la comparaison avec le mauvais fonctionnement des ordinateurs pour soutenir son raisonnement. Cela n'est pas tout à fait un argument massue, par contre, puisqu'une stricte analogie dans la distinction interne/externe entre les humains et les ordinateurs est fort peu plausible. En effet, ou bien la plupart des lecteurs auront différentes intuitions quant aux problèmes qui comptent pour des dysfonctions internes et quant aux problèmes d'ordre relationnel, ou bien ils auront très peu d'intuitions sur cette question tout court. 
Parvenus à ce point, nous pouvons réfléchir sur la méthodologie du débat. S'il doit être tranché par analyse conceptuelle, nous pouvons nous demander si nos concepts ordinaires sont définis de façon assez précise pour nous permettre de décider laquelle des analyses est correcte. Un élément qui tend à prouver que nos concepts ne sont pas suffisamment définis pour résoudre de tels débats, c'est le fait même que différentes personnes utilisent ces concepts de différentes façons. Certains théoriciens soutiennent la possibilité de troubles intrinsèquement relationnels, et on peut présumer qu'ils ne croient pas faire là un mauvais usage du concept de trouble mental. Bien sûr, il est possible qu'un groupe de théoriciens utilise les concepts correctement alors qu'un autre les utilise de façon incorrecte, mais il est difficile de voir comment la seule analyse conceptuelle fournira une méthode pour décider de l'usage correct. Notez que Wakefield affirme non seulement que sa théorie de la dysfonction préjudiciable fournit une analyse conceptuelle des troubles mentaux, mais aussi qu'elle possède un plus grand pouvoir explicatif que toute autre analyse rivale (1997, p. 269-70). Étrangement, bien qu'il ait consacré son article de 1997 à la psychopathologie développementale, Wakefield ne fournit pas d'argument pour soutenir que son approche possède un plus grand pouvoir explicatif qu'un modèle de la maladie mentale qui admet des troubles mentaux intrinsèquement relationnels.

\section{Les maladies,}

Bien que, jusqu'à maintenant, la discussion se soit concentrée sur la notion de dysfonction préjudiciable de Wakefield, on peut l'étendre à d'autres définitions des troubles mentaux. Pour en donner ne serait-ce qu'un exemple, prenons les travaux de Gert et de ses collègues, qui ont influencé la définition des troubles mentaux que l'on retrouve dans les éditions récentes du DSM. Dans une de leurs définitions les plus récentes, ces auteurs (Gert et al., 1997, p. 104) écrivent : "Un individu a une maladie si et seulement s'il est dans une condition, autre que ses croyances ou désirs rationnels, telle qu'il souffre, ou est soumis à une augmentation non triviale du risque de souffrir, d'un préjudice ou d'un mal non trivial (mort, douleur, incapacité, perte de liberté, ou perte de plaisir) en l'absence d'un soutien causal distinct. » Cette définition établit le concept de maladie en général plutôt que le concept plus spécifique de trouble mental, et évite ainsi la question problématique de la distinction entre maladie physique et maladie mentale. Elle vise à spécifier explicitement les sortes de conséquences négatives assez sérieuses pour faire de la condition qui les cause une maladie. En insistant sur le fait que la condition ne peut pas simplement consister en des croyances et désirs rationnels, la définition exclut certaines possibilités indésirables comme la médicalisation des croyances politiques. En excluant tout soutien causal distinct, elle requiert en plus que la condition soit en un certain sens intérieure à la personne, donc que les cas de souffrance tels que se faire tordre le bras par une autre personne ne soient pas considérés comme des maladies. Bien qu'elle soit plutôt vague, cette 
définition réussit au moins à embrasser plusieurs cas que l'on considérerait normalement comme des maladies, des maladies et des affections. Cependant elle engendre des difficultés importantes lorsqu'on voit comment elle s'acquitte des maladies mentales des enfants.

Compte tenu des buts que nous poursuivons, nous pouvons nous concentrer sur la stipulation qu'un individu n'est dans une condition de souffrance qu'en l'absence de lien causal distinct. Supposons qu'un enfant souffre de dépression parce que ses parents sont distants émotionnellement. Il peut y avoir plusieurs façons d'expliquer la connexion entre la condition mentale de l'enfant et le comportement de ses parents - par exemple en utilisant la théorie de l'apprentissage social, la théorie de l'attachement et même le béhaviorisme ; cela ne devrait pas changer grand chose à la définition exacte de cette connexion, dans la mesure où il y en a une. Ce genre de cas peut servir de contre-exemple possible à la définition que Gert et ses collègues donnent d'une maladie, et ceux qui la défendent pourraient alors utiliser un genre de réponse semblable à celle qu'utilise Wakefield lorsqu'il défend son point de vue. Ils pourraient insister sur le fait que, si la théorie relationnelle de la cause de la dépression est correcte, elle ne devrait pas compter pour un trouble mental mais pourrait plutôt être classée dans la catégorie plus large des problèmes de santé mentale.

Si la psychiatrie devait s'approprier les problèmes de dysfonctions individuelles et les problèmes intrinsèques plutôt que les problèmes relationnels, il est évident que cela mènerait à une plus grande divergence entre la pédopsychiatrie et les autres formes de psychologie et de travail social orientés vers les enfants. Cela s'accorderait bien avec les arguments de ceux qui prônent l'intégration de la psychiatrie avec la neurologie, les neurosciences et la génétique (Martin, 2002 ; Guze, 1992). Ces arguments reposent généralement sur le présupposé qu'un tel changement entraînerait la psychologie vers plus de scientificité et faciliterait donc les progrès dans ce champ. Notez tout de même que, dans le cas de la pédopsychiatrie, cela réduirait aussi beaucoup la portée de la discipline. Étant donné qu'une grande partie des experts dans ce champ mettent l'accent sur les facteurs relationnels dans la plupart des problèmes psychologiques de l'enfance, limiter la pédopsychiatrie aux cas qui peuvent être expliqués par un modèle de troubles neurologiques impliquerait de reléguer la majeure partie des cas à d'autres branches de la psychologie clinique. Plusieurs, dans le champ de la pédopsychiatrie, pourraient mettre en question la sagesse d'une telle approche de la profession. De plus, la nécessité de restreindre la psychiatrie aux seules dysfonctions cérébrales pour la rendre plus scientifique est loin d'être évidente. Dans le champ de la psychologie clinique, la plupart considèrent leurs recherches comme faisant partie du domaine de la science, même lorsqu'ils ne conceptualisent pas les problèmes psychologiques comme des dysfonctions internes. En effet, certains soutiennent qu'une approche complètement réductionniste visant à appréhender le mental par la psychiatrie est incorrecte pour des raisons de principes (Perring, 1999; 
Zachar, 2000), suggérant ainsi que la distinction supposée entre les approches psychologiques et neurologiques des troubles mentaux est fondamentalement erronée.

\section{Les « problèmes de santé mentale »}

Le désaccord entre Wakefield, d'un côté, Murphy et Woolfolk, de l'autre, porte sur l'étendue du concept de trouble mental. Ce désaccord pourrait se résoudre avec l'approche de Bolton (2000) qui se concentre lui aussi principalement sur la théorie de la dysfonction préjudiciable de Wakefield. Il se penche d'abord sur les arguments soutenant qu'elle est trop restrictive, parce que les troubles mentaux peuvent impliquer d'autres facteurs, tels que le mauvais appariement entre la constitution [design] de l'organisme et l'environnement, mais aussi certaines caractéristiques évoluées de la configuration [design] de l'être humain, ainsi que des stratégies défensives et d'autres qui impliquent la perturbation d'une fonction $(2000$, p. 146). Bolton examine comment Wakefield traite ce genre de cas et décrit la façon dont il défend son point de vue : en insistant sur le fait qu' "en l'absence de dysfonction, définie en termes de défaillance structurelle [design], il ne peut ni ne doit y avoir attribution de trouble» (2000, p. 148). Puisque Bolton est particulièrement préoccupé par les symptômes associés aux troubles mentaux, il n'est pas nécessairement enthousiaste à l'idée que des comportements problématiques soient considérés comme des troubles mentaux et il accueille donc favorablement la conclusion de Wakefield. Il soutient que les gens peuvent éprouver des problèmes et être traités par des cliniciens même si ces problèmes ne sont pas considérés comme des troubles mentaux, et il explique à quel point il est difficile de trouver un nom adéquat pour cette catégorie de problèmes à cause des connotations problématiques de tous les candidats principaux (2000, p. 149). Il arrête son choix sur le terme assez large de "problèmes de santé mentale » qui inclut à la fois les troubles mentaux, les problèmes causés par le mauvais appariement entre la configuration [design] de l'organisme et l'environnement, les stratégies défensives et le reste. Bolton soutient que, puisque notre connaissance de la psychologie évolutionniste est si limitée, nous savons peu de choses quant à ce qui devrait être considéré comme un trouble mental, bien que nous ayons une bonne idée de ce qu'est un problème de santé mentale. Il souligne qu'il y a des avantages et des inconvénients à classer les conditions comme troubles mentaux ou problèmes de santé mentale, mais je n'exposerai pas ici le détail de ses arguments. Étant donné nos objectifs, le point essentiel est que la suggestion de Bolton est entièrement compatible avec une conception intrinsèquement relationnelle des problèmes de santé mentale. Néanmoins, comme il est peu disposé à étendre la catégorie des troubles mentaux, puisque des symptômes y sont rattachés, Bolton serait probablement réticent à embrasser le concept de troubles mentaux intrinsèquement relationnels. Son raisonnement ne serait pas basé sur quelque scrupule quant à l'incohérence du concept, mais plutôt sur des considérations pragmatiques. 
La discussion de Bolton soulève la question de savoir pourquoi on doit tracer une distinction entre les conditions considérées comme des troubles et celles qui ne le sont pas. Sa suggestion de faire sans le concept de trouble est attrayante sous plusieurs aspects, et elle pourrait résoudre un bon nombre de problèmes théoriques et pratiques. C'est une suggestion radicale qui nécessiterait de nombreux changements sociaux, puisque beaucoup de nos pratiques, tout particulièrement les pratiques morales concernant la responsabilité de nos actions, et les décisions financières, à savoir qui mérite de l'aide, dépendent d'une distinction entre ceux qui sont autonomes et qui contrôlent leur comportement et ceux dont la condition les fait agir de façon à se nuire à euxmêmes [in self-defeating ways] ou souffrir d'émotions qui les empêchent de participer pleinement. Bien que, dans le domaine légal, la tendance dans les sociétés occidentales consiste à insister sur le fait que les contrevenants doivent souffrir de psychose majeure pour pouvoir être acquittés pour cause d'aliénation mentale, nous sommes plutôt prêts à accepter que les gens devraient être moins blâmés moralement si leur incompétence ou leur mauvais comportement résulte d'une maladie mentale. Nous n'avons pas tendance à blâmer, par exemple, ceux qui agissent sans inhibition lorsque cela résulte d'un accident cérébral ou de problèmes neurologiques. De même, lorsque ceux qui sont atteint de manies s'endettent terriblement ou causent des problèmes par leur promiscuité sexuelle, on ne tend pas à les blâmer pour leur immoralité, mais plutôt à voir leurs actions comme des symptômes de leur maladie. Le scepticisme à propos de l'utilisation d'un trouble mental comme excuse a généralement pour cause un scepticisme concernant la question de savoir si le comportement problématique est réellement le résultat d'une condition médicale. Si nous adoptions la suggestion de Bolton et que nous arrêtions de parler de maladie mentale pour adopter plutôt la catégorie plus large de problème de santé mentale, il faudrait minimalement repenser ce qui compte comme excuse pour un mauvais comportement, et il est probable que nous devrions apporter à nos pratiques des changements importants.

Cette question n'est peut-être pas aussi importante pour les enfants, puisqu'ils n'ont pas atteint leur pleine maturité et que, de toute façon, on ne les tient pas responsables de leurs actions. De plus, nous avons tendance à penser que les enfants dans le besoin devraient recevoir de l'aide quelle que soit la cause de leurs problèmes, et nous n'avons donc pas autant besoin d'établir une distinction entre problèmes causés par soi-même et troubles chez les enfants. Néanmoins, la question de savoir quand tenir les gens responsables de leurs actions ne disparaît pas pour autant lorsqu'on considère le cas des enfants et des adolescents. En effet, un des objectifs principaux de l'éducation des enfants est de les amener à assumer la responsabilité de leur comportement et à faire partie d'une communauté qui tient les gens responsables de leurs actions. Une partie essentielle de cette pratique est la maîtrise de l'habileté à distinguer les cas où les gens sont responsables de leurs actions de ceux où ils ne le sont pas. 


\section{Les critères d'une définition adéquate des troubles mentaux}

Malgré tout, si l'on exclut la perspective que les choses tournent de façon à ce qu'on en vienne à se passer du concept de trouble mental, on a besoin de la meilleure définition que l'on puisse trouver. Il pourrait être utile, à ce stade-ci du débat, de se pencher sur les critères selon lesquels nous devrions juger qu'une définition des troubles mentaux est adéquate. Nous voulons qu'elle s'accorde avec nos intuitions pré-théoriques quant à ce qui compte pour un trouble, qu'elle puisse être utilisée pour autoriser le traitement approprié de la souffrance (tout spécialement lorsque la responsabilité du système de santé ou d'un tiers comme bailleur de fonds est en jeu), qu'elle soit utile dans un contexte légal, ainsi que pour la recherche scientifique, qu'elle s'accorde avec nos meilleures théories scientifiques, et finalement qu'elle facilite la communication entre les professionnels de la santé. Suivant la suggestion de Bolton, nous pourrions aussi favoriser une définition qui ne renforce pas les symptômes associés aux troubles mentaux. Ces différents desiderata pourraient fort bien nous pousser dans des directions différentes, et nous pourrions bien devoir leur assigner une importance différente pour pouvoir décider de la meilleure définition. Nous pourrions aussi simplement utiliser différentes définitions et différentes nosologies pour divers objectifs, plutôt que d'utiliser un seul système de classification ; en effet, les politiques de santé et d'éducation du gouvernement et de la loi utilisent parfois des critères différents pour distinguer les cas qui demandent une attention spéciale pour des raisons de santé.

Il vaut la peine d'insister sur le fait que la pédopsychiatrie pourrait accorder à tous ces facteurs une importance différente de celle que leur accordent les autres sous-disciplines de la psychiatrie; on peut soutenir, entre autres, comme nous l'avons noté précédemment, qu'elle a donné moins d'importance à la rigueur dans le diagnostic et davantage à l'aide apportée au patient individuel que ne l'a fait la psychiatrie des adultes. Ainsi, suivant cette tradition, il est plausible que la pédopsychiatrie soit moins préoccupée par la formulation d'une définition stricte des troubles mentaux et qu'elle puisse se satisfaire de la catégorie plus large de problème de santé mentale de Bolton, puisque le plus important est de fournir aux enfants de l'aide et des traitements tandis que le besoin de séparer les enfants dont les problèmes sont individuels de ceux dont les problèmes sont relationnels est moindre.

Ce qui ressort clairement de la discussion précédente, c'est que la thèse sur la vérité conceptuelle du fait que les troubles mentaux de l'enfance sont des dysfonctions individuelles est fort peu plausible. Au mieux, il s'agit là d'un concept parmi d'autres concepts afférents qui sont regroupés sous la catégorie générale des troubles mentaux de l'enfance. Il faudrait pour soutenir cette thèse un type d'argument différent, qui montrerait pourquoi l'analyse principale des troubles mentaux de l'enfance devrait s'attaquer au concept particulier de dysfonction interne, et la discussion précédente devrait à tout le moins avoir montré qu'il sera difficile de rendre cet argument convaincant. 


\section{Conclusion}

Avant que nous puissions mettre un terme au débat sur la classification des troubles mentaux des enfants et des adolescents, nous devons clarifier la fonction assignée à la nosologie. Il est clair que des considérations pragmatiques doivent être prises en considération pour régler les désaccords quant à la façon d'établir une distinction entre la normalité et la pathologie, et de créer les différentes catégories et sous-catégories de la pathologie. Néanmoins, il se peut que lorsqu'un système de classification est conçu pour atteindre divers objectifs, tels que faciliter la communication entre les chercheurs en psychiatrie, répondre aux besoins des cliniciens qui doivent décider d'un traitement approprié et fournir un système pour décider quand une assurance privée où le système de santé doit payer pour les traitements, ces divers objectifs nous poussent dans des directions différentes. Une approche pleinement pragmatique de la classification des troubles mentaux, comme celle que propose Agich (1997), pourrait pour cette raison ne pas être en mesure de fournir des critères définitifs.

Les difficultés psychologiques des enfants fournissent un excellent exemple de la question de savoir si une dysfonction interne chez un individu est la condition nécessaire d'un trouble mental, et la question connexe est de savoir ce qui doit être considéré comme une dysfonction interne. Ce survol des différentes approches suggère que l'analyse conceptuelle et la théorie psychiatrique ne peuvent elles-mêmes fournir de critères neutres et précis pour les troubles mentaux. Bien qu'on voie facilement l'attrait de restreindre le concept de troubles mentaux aux cas de dysfonction identifiable chez un individu, il serait prématuré de conclure que c'est la seule approche viable. Il est urgent d'examiner philosophiquement la cohérence du concept de troubles psychiatriques chez les couples et les familles ainsi que l'élaboration du rapport entre de tels troubles et les troubles relationnels des individus. Ma thèse centrale dans cet article n'est pas que l'on doit accepter la réalité que les troubles mentaux intrinsèquement relationnels sont imputables aux couples et aux familles plutôt qu'aux individus, mais qu'il pourrait nous être utile d'adopter une telle conceptualisation, et l'on ne devrait pas écarter a priori cette possibilité en soutenant faussement comme vérité conceptuelle inéluctable que les troubles mentaux ne peuvent être attribués qu'aux individus.

(Traduit de l'anglais par Nicolas Payette)

\section{Bibliographie}

Agich, G.J. "Toward a Pragmatic Theory of Disease ", dans J.M. Humber et R.F. Almeder, dir., What is Disease ?, Totowa, N.J., Humana Press, 1997, p. 219 246.

Bolton, D. «Alternatives to Disorder », Philosophy, Psychiatry, \& Psychology, 7, 2, 2000, p. 141-153.

Borduin, C.M. et al. "Relational Problems : The Social Context of Child and Adolescent Disorders ", dans S.D. Netherton et al., Child and Adolescent 
Psychological Disorders : A Comprehensive Textbook, New York, Oxford University Press, 1999, p. 498-519.

Cummings, E.M., P.T. Davies, et S.B. Campbell. Developmental Psychopathology and Family Process: Theory, Research, and Clinical Implications, New York, Guilford Press, 2000.

De Grandpré, R. Ritalin Nation: Rapid-Fire Culture and the Transformation of Human Consciousness, New York, W.W. Norton, 1999.

Douchette, A. "Child and Adolescent Diagnosis : The Need for a Model-Based Approach ", dans L.E. Beutler et M.L. Malik, Rethinking the DSM : A Psychological Perspective, Washington, D.C., American Psychological Association, 2002, p. 201-220.

Gert, B. et al. Bioethics : A Return to Fundamentals, New York, Oxford University Press, 1997.

Guze, S.B. Why Psychiatry is a Branch of Medicine, New York, Oxford University Press, 1992.

Henggler, S.W. et C.M. Borduin. Family Therapy and Beyond: A Multisystemic Approach to Treating the Behavior Problems of Children and Adolescents, Pacific Grove, CA, Brooks/Cole Publishing Company, 1999.

Jensen, P.S. et K. Hoagwood. "The Book of Names : DSM-IV in Context ", Development and Psychopathology, 19, 1997, p. 231-249.

Hudziak, J.J. "Importance of Phenotype Definition in Genetic Studies of Child Psychopathology », dans J.E. Helzer et J.J. Hudziak, Defining Psychopathology in the $21^{\text {st }}$ Century : DSM-V and Beyond, Washington, D.C., American Psychiatric Association, 2002, p. 211-230.

Jensen, P.S. et les membres du MTA Cooperative Group. « ADHD Comorbidity Findings From the MTA Study : New Diagnostic Subtypes and Their Optimal Treatments », dans J.E. Helzer et J.J. Hudziak, Defining Psychopathology in the $21^{\text {st }}$ Century : DSM-V and Beyond, Washington, D.C., American Psychiatric Association, 2002, p. 169-192.

Marin, J.B. "The Integration of Neurology, Psychiatry, and Neuroscience in the 21st Century ", American Journal of Psychiatry, 159, 9, 2002, p. 695-704.

Murphy, D. et R.L. Woolfolk. "The Harmful Dysfunction Analysis of Mental Disorder ", Philosophy, Psychiatry \& Psychology, 7, 4, 2000, p. 241-252.

Perring, C. "The Neuron Doctrine in Psychiatry », commentaire de I. Gold et D. Stoljar, "A Neuron Doctrine in the Philosophy of Neuroscience ", Behavioral and Brain Sciences, 22, 1999.

—. "Mental Illness ", dans E.N. Zalta, dir.,

The Stanford Encyclopedia of Philosophy (Spring 2002 Edition), [http ://plato.stanford.edu/archives/spr2002/entries/mental-illness/].

Reiss, D. et R.N. Emde. «Relationship Disorders Are Psychiatric Disorders : Five Reasons They Were Not Included in DSM-IV », dans K. Phillips et al., Advancing DSM : Dilemmas in Psychiatric Diagnosis, Washington, D.C., America Psychiatric Association, 2002.

Schowalter, J. « Child and Adolescent Psychiatry Comes of Age, 1944-1994 ", dans R.W. Menninger et J.C. Nemiah, dir., American Psychiatry After World War II 1944-1994, Washington, D.C., American Psychiatric Press, 2000, p. 461-480.

U.S. Department of Health and Human Services, Mental Health: A Report of the Surgeon General, Rockville (MD), U.S. Department of Health and Human Services, Substance Abuse and Mental Health Services Administration, Center 
for Mental Health Services, National Institutes of Health, National Institute of Mental Health, 1999.

Wakefield, J.C. "When is development disordered ? Developmental psychopathology and the harmful dysfunction analysis of mental disorder ", Development and Psychopathology, 9, 1997, p. 269-290.

—. "Spandrels, Vestigial Organs, and Such ", Philosophy, Psychiatry, \& Psychology, 7, 4, 2002, p. 253-269.

World Health Organization, International Classification of Disease (Tenth Edition) ICD-10, Geneva, World Health Organization, 1992.

Zachar, P. Psychological Concepts and Biological Psychiatry, Amsterdam, John Benjamins, 2000. 